\title{
Radiation-induced parotid changes in oropharyngeal cancer patients: the role of early functional imaging and patient -/treatment-related factors
}

Simona Marzi ${ }^{1 *}$ D, Alessia Farneti ${ }^{2}$, Antonello Vidiri ${ }^{3}$, Francesca Di Giuliano ${ }^{3,4}$, Laura Marucci ${ }^{2}$, Filomena Spasiano ${ }^{2}$, Irene Terrenato ${ }^{5}$ and Giuseppe Sanguineti ${ }^{2}$

\begin{abstract}
Background: Functional magnetic resonance imaging may provide several quantitative indices strictly related to distinctive tissue signatures with radiobiological relevance, such as tissue cellular density and vascular perfusion. The role of Intravoxel Incoherent Motion Diffusion Weighted Imaging (IVIM-DWI) and Dynamic Contrast-Enhanced (DCE) $\mathrm{MRI}$ in detecting/predicting radiation-induced volumetric changes of parotids both during and shortly after (chemo) radiotherapy of oropharyngeal squamous cell carcinoma (SCC) was explored.

Methods: Patients with locally advanced oropharyngeal SCC were accrued within a prospective study offering both IVIM-DWI and DCE-MRI at baseline; IVIM-DWI was repeated the 10th fraction of treatment. Apparent diffusion coefficient (ADC), tissue diffusion coefficient $D_{t}$, perfusion fraction $f$ and perfusion-related diffusion coefficient $D^{*}$ were estimated both at baseline and during RT. Semi-quantitative and quantitative parameters, including the transfer constant $K^{\text {trans }}$, were calculated from DCE-MRI. Parotids were contoured on T2-weighted images at baseline, 10th fraction and 8th weeks after treatment end and the percent change of parotid volume between baseline/10th $\operatorname{fr}\left(\Delta \mathrm{Vol}_{10 \mathrm{fr}}\right)$ and baseline/8th wk. ( $\left.\Delta \mathrm{Vol}_{\text {post }}\right)$ computed.

Correlations among volumetric changes and patient-, treatment- and imaging-related features were investigated at univariate analysis (Spearman's Rho).

Results: Eighty parotids (40 patients) were analyzed. Percent changes were $18.2 \pm 10.7 \%$ and $31.3 \pm 15.8 \%$ for $\Delta V_{\mathrm{O}} \mathrm{I}_{1 \text { ofr }}$ and $\Delta \mathrm{Vol}_{\text {post, }}$ respectively. Among baseline characteristics, $\Delta \mathrm{Vol}_{1 \text { ofr }}$ was correlated to body mass index, patient weight as well as the initial parotid volume. A weak correlation was present between parotid shrinkage after the first 2 weeks of treatment and dosimetric variables, while no association was found after radiotherapy. Percent changes of both $A D C$ and $D_{t}$ at the 10th fraction were also correlated to $\left.\Delta V_{0}\right|_{1 \text { ofr. }}$ Significant relationships were found between $\triangle V_{\text {ol }}$ post and baseline DCE-MRI parameters.

Conclusions: Both IVIM-DWI and DCE-MRI can help to detect/predict early (during treatment) and shortly after treatment completion the parotid shrinkage. They may contribute to clarify the correlations between volumetric changes of parotid glands and patient-/treatment-related variables by assessing individual microcapillary perfusion and tissue diffusivity.
\end{abstract}

Keywords: Diffusion magnetic resonance imaging, Perfusion magnetic resonance imaging, Radiotherapy, Parotid gland, Oropharyngeal Cancer

\footnotetext{
* Correspondence: simona.marzi@ifo.gov.it

${ }^{1}$ Medical Physics Laboratory, IRCCS Regina Elena National Cancer Institute,

Via Elio Chianesi 53, 00144 Rome, Italy

Full list of author information is available at the end of the article
}

(c) The Author(s). 2018 Open Access This article is distributed under the terms of the Creative Commons Attribution 4.0 International License (http://creativecommons.org/licenses/by/4.0/), which permits unrestricted use, distribution, and reproduction in any medium, provided you give appropriate credit to the original author(s) and the source, provide a link to the Creative Commons license, and indicate if changes were made. The Creative Commons Public Domain Dedication waiver (http://creativecommons.org/publicdomain/zero/1.0/) applies to the data made available in this article, unless otherwise stated. 


\section{Background}

Radiotherapy is involved in the treatment of the majority of patients with non-metastatic head and neck squamous cell carcinoma [1]. Despite intensity modulated radiotherapy (IMRT), xerostomia is still a frequent radiation-induced complication with implications on patients quality of life [2, 3]. More importantly, there is some degree of inconsistency between dosimetric data of the parotids at planning and long-term patient reported outcomes [4]. Among possible explanations, variations in intrinsic radiosensitivity among individuals [5], discrepancies between objective outcome and patient perception [6] and variations in parotid gland (PG) dosage during the course of IMRT due to shrinkage have been advocated $[7,8]$.

Thought the pathophysiology of parotid shrinkage during radiotherapy remains unclear [9], image-based scoring of toxicity based on early changes of irradiated organs can provide a timely, objective and consistent endpoint for patient counseling and treatment strategy adaptation [5, 10-12]. In this regard, functional magnetic resonance imaging (MRI) techniques, such as diffusion- and perfusion- MRI, may have an important role, as they provide several quantitative indices strictly related to distinctive tissue signatures with radiobiological relevance, such as tissue cellular density and vascular perfusion $[12,13]$.

Dynamic contrast-enhanced (DCE) MRI is a wellestablished technique to evaluate the hemodynamic properties of several tissues, such as vessel leakiness and permeability, by means of serial MRI scans taken before and after the intravenous injection of a contrast agent [13, 14]. Moreover, diffusion-weighted imaging (DWI) provides insights into cellular architecture, being sensitive to the thermally driven motion of water molecules in tissues [15]. Therefore, DWI indirectly assesses cell depopulation due to radiation-induced changes. Intravoxel Incoherent Motion (IVIM) imaging is a further evolution of DWI potentially able to provide both diffusion and perfusion imaging results with the additional advantage of being completely non-invasive, as it does not require contrast injection $[16,17]$. IVIM-DWI assumes that the microcirculation of blood can be thought as a pseudo-diffusion incoherent motion, because the capillary network does not have a specific spatial orientation within a single image voxel. To separate the perfusion effects from those of thermal diffusion, IVIM-DWI requires the acquisition of images with multiple $b$ value, where $b$ is $a$ factor dependent on the gradient pulse sequence (i.e. pulse duration and strength of the diffusion gradient). The perfusion effect on the signal attenuation may be significant at low b values $(0-150 \mathrm{~s} / \mathrm{mm} 2)$ in well-vascularized tissues, while it is assumed to vanish at higher $b$ values due to the larger drop in signal compared to the thermal diffusivity effect. By using a bi-exponential function to model the IVIM signal intensity at increasing b values [16], both perfusionrelated and perfusion-free diffusion parameters may be derived, as described below in more detail.

In January 2016 we started a prospective study funded by the Italian Association for Cancer Research (AIRC, project No.17028) in locally advanced oropharyngeal SCC investigating the ability of both DCE-MRI and IVIM-DWI to predict tumor response to (chemo-)IMRT. As part of this ongoing study, we investigated also volumetric changes of parotid glands during and shortly after treatment completion. Here we report the results on 40 consecutive patients.

\section{Methods}

\section{Patient population\& treatment}

To be eligible, patients had to fulfill all the following criteria: (i) age older than 18 years; (ii) Karnofsky performance status > 80; (iii) pathologically confirmed squamous cell carcinoma of the oropharynx; (iv) stage III or IV without distant metastases according to the 7th edition of the AJCC Cancer Staging Manual; (v) treatment with radiotherapy \pm chemotherapy. Exclusion criteria included: any contraindication for MR examination; prior surgery, CHT (including induction chemotherapy) or radiotherapy to the primary disease and the neck. Moreover, specific informed consent was obtained from each patient before enrolling. The study protocol was approved by the local Institutional Review Board.

All patients were offered IMRT and concomitant cisplatin (CDDP), either $100 \mathrm{mg} / \mathrm{m}^{2}$ for three cycles every 21 days or weekly $40 \mathrm{mg} / \mathrm{m}^{2}$ for 6 cycles. If patients were not medically fit, IMRT alone was delivered. A simultaneous integrated boost technique delivering the following total doses in 35 fractions was used: 70 Gy to macroscopic disease; 63 Gy to regions at high risk of microscopic disease; 58.1 Gy to regions at intermediate risk of microscopic disease [18]. A 7-field approach with $6 \mathrm{MV}$ photons was used. For each patient, the planned mean dose to the single parotid gland, $\mathrm{D}_{\text {mean }}(\mathrm{Gy})$, and the percentage of parotid volume receiving $\geq 30 \mathrm{~Gy}, \mathrm{~V}_{30}(\%)$, were calculated from the treatment planning system (Eclipse version 13.5.37, Varian Medical System, Palo Alto, CA) and recorded.

\section{MR imaging protocols}

MRI scans were performed with a $1.5-\mathrm{T}$ system (Optima MR 450w, GE Health-care, Milwaukee, Wisconsin) with dedicated 16-channels receive-only RF coils: a head coil, a surface neck and a spine coil. In addition to standard imaging, all patients underwent three serial MRI examinations: before treatment, after the 10th fraction of chemoradiotherapy and 8 weeks after the end of treatment. MRI scanning was avoided on the same day of chemotherapy administration. 
At baseline, the MRI acquisition protocol included both IVIM-DWI and DCE-MRI examinations. However, only IVIM-DWI was repeated at the 10th fraction to reduce the use of intravenous contrast agent. Both coronal and axial fast spin echo $\mathrm{T}_{2}$-weighted images (acquisition matrix, $288 \times 256$; field of view, $26-28 \mathrm{~cm}$; TR/TE, 5739/100 ms; slice thickness, $4 \mathrm{~mm}$; inter-slice gap, 5 ; acquisition time, 2 min and 40 s) were acquired in all MRI exams.

IVIM-DWI was performed using single-shot echo-planar imaging with the following parameters: acquisition matrix $128 \times 128$, field of view $26-28 \mathrm{~cm}$, TR/TE $4500 \mathrm{~ms} / 77 \mathrm{~ms}$, number of slices 30-33, slice thickness $4 \mathrm{~mm}$, and intersection gap $1 \mathrm{~mm}$. Nine different $b$ values $(b=0,25,50,75$, $100,150,300,500$, and $800 \mathrm{~s} / \mathrm{mm}^{2}$ ) were used, with all diffusion-sensitizing gradients applied in three orthogonal directions to obtain trace-weighted images.

To enable the perfusion-sensitive information to be accurately extracted from DWI, most of the b values (six) were chosen within the lower range $0-150 \mathrm{~s} / \mathrm{mm}^{2}$, while three $b$ values in the range $300-800 \mathrm{~s} / \mathrm{mm}^{2}$ were considered adequate to estimate the perfusion-free tissue diffusion coefficient. The maximum $b$ value was limited to $800 \mathrm{~s} / \mathrm{mm}^{2}$ as it usually provides a sufficient signal to noise ratio and a good lesion cospicuity in the head and neck region at 1.5-T.

Three signal averages were chosen for $b$ values ranging from 0 to $300 \mathrm{~s} / \mathrm{mm}^{2}$, four for a $b$ value of $500 \mathrm{~s} /$ $\mathrm{mm}^{2}$, and five for a $b$ value of $800 \mathrm{~s} / \mathrm{mm}^{2}$. Array Spatial Sensitivity Encoding Technique imaging, with a scantime reduction factor of 2 , was used, with a resulting scanning time of 6 min and $13 \mathrm{~s}$.
DCE-MRI involved a 3D fast-spoiled gradient echo sequence with the following characteristics: TR/ TE:3.82/1.18 ms; flip angle: $30^{\circ}$; acquisition matrix: $128 \times 128$; field of view: $28 \mathrm{~cm}$; number of slices: 32 ; slice thickness: $4 \mathrm{~mm}$; spacing between slices: $2 \mathrm{~mm}$.

Sixty dynamic volumes were acquired consecutively, with a temporal resolution of $5 \mathrm{~s}$ and a total scanning time of $5 \mathrm{~min}$. At the fourth dynamic volume, $0.1 \mathrm{mmol} / \mathrm{kg}$ body weight of gadopentetatedimeglumine contrast agent was administered intravenously, at a rate of $3 \mathrm{ml} / \mathrm{s}$.

\section{Parotid gland delineation}

For each patient, a single observer manually outlined the entire PG on T2-weighted images acquired at each time point. 3D Slicer Software (version 4.6.2) was used for image visualization and for gland segmentation [19]. Vascular structures were meticulously excluded from the delineated PG volume. These contours were propagated onto DCE-MRI images using the 3D Slicer module Expert Automated Registration.

The parotid contours obtained on T2-weighted images also served as a guide to parotid gland delineation on DWI sequences with $b=800 \mathrm{~s} / \mathrm{mm}^{2}$. An example of parotid delineation is illustrated in Fig. 1.

\section{Calculation of IVIM-diffusion parameters}

Dedicated scripts were developed in Matlab code (Release 7.10.0, The Mathworks Inc., Natick, Massachusetts) for quantitative image analyses. The median value of the signal from all voxels within the entire gland was derived
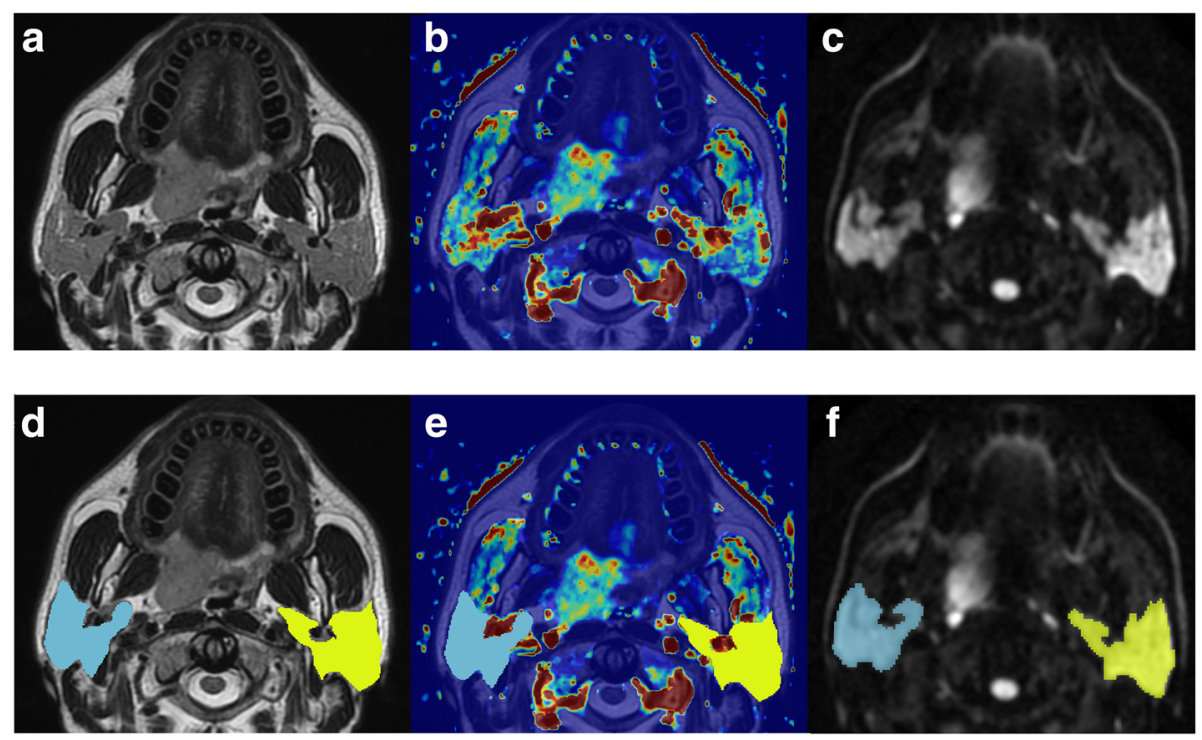

Fig. 1 Central section of parotids in a 44-year-old man affected by a tonsil carcinoma on the axial T2-weighted image (a), on the map of $\mathrm{K}^{\text {trans }}$ (b) and on the diffusion-weighted image obtained with b of $800 \mathrm{~s} / \mathrm{mm}^{2}$ (c) in the same anatomic location. Bottom: user-defined parotid contours overlaid on the axial T2-weighted image (d) and on the corresponding map of $\mathrm{K}^{\text {trans }}(\mathbf{e})$ and diffusion-weighted image obtained with $\mathrm{b}$ of $800 \mathrm{~s} / \mathrm{mm}^{2}(\mathbf{f})$ 
for each $b$ value and its variation at increasing $b$ was modeled using the following bi-exponential function by a nonlinear constrained minimization algorithm [16]: $\mathrm{S}_{\mathrm{b}} / \mathrm{S}_{0}=(1-\mathrm{f}) \cdot \mathrm{e}^{-\mathrm{b} \cdot \mathrm{D}}+\mathrm{f} \cdot \mathrm{e}^{-\mathrm{b} \cdot\left(\mathrm{D}+\mathrm{D}^{*}\right)}$ where $S_{b}$ is the signal intensity with diffusion weighting $b, S_{0}$ is the signal intensity for a $b$ value of $0 \mathrm{~s} / \mathrm{mm}^{2}, f$ is the fractional volume of capillary blood, $D_{t}$ is the tissue diffusion coefficient (in $\mathrm{mm}^{2} / \mathrm{s}$ ), and $\mathrm{D}^{*}$ is the perfusion-related diffusion coefficient (in $\mathrm{mm}^{2} / \mathrm{s}$ ).

As described elsewhere [20], a 'two step' fitting method was implemented to avoid overfitting: firstly the $\mathrm{D}_{t}$ value was derived from data at $b$ values of 300,500 and $800 \mathrm{~s} /$ $\mathrm{mm}^{2}$ using a mono-exponential fit; secondly fixing $\mathrm{D}_{t}$ at the value estimated above, $\mathrm{S} 0, \mathrm{D}^{*}$, and $f$ were determined according to Eq.1. To avoid the risk of suboptimal solutions, one hundred simultaneous optimization processes were launched for each fit, starting from randomly chosen initial values of parameters between fixed bounds. The residual sum of squares value of each optimization process was recorded and the minimum value was used to assess the best solution for the parameters.

The conventional ADC was also derived from data at $b$ values of 0,500 and $800 \mathrm{~s} / \mathrm{mm}^{2}$, using a mono-exponential model [15].

\section{Calculation of the DCE-MRI parameters}

The DCE-MRI data were analyzed using a commercial software package (GenIQ General, GE Advanced Workstation, Palo Alto, CA). The pharmacokinetic analysis was performed based on the Toft model: the quantitative parameters $\mathrm{K}^{\text {trans }}$, the transfer constant between plasma and the extravascular extracellular space (EES), $\mathrm{K}_{\mathrm{ep}}$, the transfer constant between EES and plasma and $\mathrm{v}_{\mathrm{e}}$, the fractional volume of EES were calculated [14]. A semi-quantitative parameter, the initial area under gadolinium concentration curve or IAUGC (calculated from the bolus arrival to the first $90 \mathrm{~s}$ ) was also derived. The median of each parameter, computed from the distribution of the voxel-level values within the entire gland, was used for statistical purposes.

\section{Statistics}

The change in parotid volume was estimated as the percent volume reduction at both the 10th fraction of RT $\left(\Delta \mathrm{Vol}_{1 \text { frr }}\right)$ and the 8 th week after the end of IMRT $\left(\Delta \mathrm{Vol}_{\text {post }}\right)$.

The Saphiro-Wilk test was used to test the normality of the data. As some of the variables (about 49\%) were not normally distributed, non-parametric analyses were performed, as they are based on distribution-free methods. The Wilcoxon test for paired samples was used to assess whether changes over time were significant. Correlations among volumetric changes at the time points and various patient-, treatment- and imaging-related features were investigated at univariate analysis with the Spearman test. We did not apply any adjustment to the $p$-values for multiple comparisons because we have only tested multiple variables within a single group and not between groups. A p level $<0.05$ was considered to indicate statistical significance. SPSS software (SPSS version 21, SPSS Inc., Chicago, IL, USA) was used for the statistical analyses.

\section{Results}

Forty patients/80 parotids were accrued and analyzed. Selected patient and tumor characteristics are summarized in Table 1. All patients received the planned radiotherapy dose and all but 7 patients received concomitant chemotherapy as well. The average $( \pm S D)$ patient weight loss at the 10th fraction and at the end of RT were $2.0 \pm 2.7 \mathrm{Kg}$ $(-2.7 \% \pm 3.8 \%)$ and $6.8 \pm 4.4 \mathrm{Kg}(9.0 \% \pm 5.1 \%)$, respectively. At the 10th fraction, the parotid volume decreased from a mean $( \pm \mathrm{SD})$ value of $32.3( \pm 9.4) \mathrm{cm}^{3}$ to $26.7( \pm 9.0)$ $\mathrm{cm}^{3}$, with a mean $\Delta \mathrm{Vol}_{1 \text { fr }}$ of $18.2 \%( \pm 10.7 \%)$. Eight weeks after RT, it decreased to $22.7( \pm 8.9) \mathrm{cm}^{3}$, with a mean $\Delta \mathrm{Vol}_{\text {post }}$ of $31.3 \%( \pm 15.8)$; all but two patients were not evaluated at this time point as they died of progressive

Table 1 Selected patient and tumor characteristics

\begin{tabular}{|c|c|}
\hline Characteristic & Parameter \\
\hline Patient number & 40 (80 parotids) \\
\hline $\operatorname{Sex}(M / F)$ & $30 / 10$ \\
\hline \multicolumn{2}{|l|}{ Age (years) } \\
\hline Mean (range) & $65.5(46-81)$ \\
\hline \multicolumn{2}{|l|}{ Primary tumor site } \\
\hline Tonsil & $26(65 \%)$ \\
\hline Base of Tongue & $14(35 \%)$ \\
\hline \multicolumn{2}{|l|}{ T stage } \\
\hline $\mathrm{T} 1$ & $4(10 \%)$ \\
\hline $\mathrm{T} 2$ & $11(27.5 \%)$ \\
\hline T3 & 7 (17.5\%) \\
\hline T4 & $15(37.5 \%)$ \\
\hline T4a & $3(7.5 \%)$ \\
\hline \multicolumn{2}{|l|}{$\mathrm{N}$ stage } \\
\hline NO & $4(10 \%)$ \\
\hline $\mathrm{N} 1$ & $4(10 \%)$ \\
\hline $\mathrm{N} 2 \mathrm{a}$ & $2(5 \%)$ \\
\hline $\mathrm{N} 2 \mathrm{~b}$ & $15(37.5 \%)$ \\
\hline $\mathrm{N} 2 \mathrm{C}$ & $14(35 \%)$ \\
\hline N3 & $1(2.5 \%)$ \\
\hline \multicolumn{2}{|c|}{ Parotid Radiation Dose } \\
\hline$D_{\text {mean }}(G y)$ & $35.8 \pm 8.9$ \\
\hline$V_{30}(\%)$ & $53.6 \pm 19.9$ \\
\hline
\end{tabular}

Abbreviations: $D_{\text {mean }}$ Planned mean dose to the parotid gland, $V_{30}(\%)$ Percentage of parotid volume receiving a dose $\geq 30 \mathrm{~Gy}$ 
disease. No correlation was found between $\Delta \mathrm{Vol}_{10 \mathrm{fr}} / \Delta \mathrm{Vol}$ post and the patient weight loss at the 10th fraction and at the end of RT.

At baseline, the average $( \pm S D)$ values for $K^{\text {trans }}, K_{e p}, v_{e}$ and IAUGC were $0.546( \pm 0.290) \mathrm{min}^{-1}, 2.728( \pm 1.146)$ $\min ^{-1}, 0.178( \pm 0.061)$ (fractional units) and 0.287 $( \pm 0.095)$ (arbitrary units), respectively. Summary statistics of the diffusion parameters and their variations during treatment are shown in Table 2. Only the changes of ADC and $\mathrm{D}_{\mathrm{t}}$ over time were significant $(p<0.001$ for both).

\section{Correlation between parotid shrinkage and variables at baseline}

Table 3 reports the relationships between percent parotid shrinkage and various covariates at baseline. A significant correlation was found between $\Delta \mathrm{Vol}_{10 \mathrm{fr}}$ and initial parotid volume, patient weight, $B M I, D_{\text {mean }}$ and $V_{30}$. Scatter plots of parotid shrinkage versus $D_{\text {mean }}$ and $V_{30}(\%)$ are depicted in Fig. 2. Among image-related parameters, only $K_{\text {ep }}$ was weakly associated to parotid shrinkage ( $\mathrm{Rho}=0.239$, $p=0.038)$ while $\mathrm{K}^{\text {trans }}$ showed only a trend towards statistical significance (Rho $=0.210, p=0.069$ ).

Interestingly, none of the selected patient- and treatmentrelated variables was correlated to percent shrinkage at 8 weeks after treatment completion while several DCEMRI-related parameters showed a moderate correlation at this time point, with stronger evidence (smaller significant $p$ values) than at the 10 th fraction (Table 3 ).

\section{Correlation between parotid shrinkage and diffusion parameters during treatment}

The correlation between parotid shrinkage and DWIIVIM parameters at the 10th fraction are reported in Table 4. $\Delta$ Vol $_{10 \text { fr }}$ significantly correlated with $\mathrm{D}_{\mathrm{t}}$ and the percent change of both $\mathrm{ADC}$ and $\mathrm{D}_{t}$ between baseline and the 10th fraction.

Table 2 Summary statistics of the diffusion parameters and their variations during treatment

\begin{tabular}{lllll}
\hline Parameter $^{a}$ & before RT & at the 10th & $\begin{array}{l}\text { Variation from } \\
\text { baseline (\%) }\end{array}$ & $p$ \\
\hline $\begin{array}{l}\text { ADC } \\
\left(10^{-3} \mathrm{~mm}^{2} / \mathrm{s}\right)\end{array}$ & $1.275 \pm 0.158$ & $1.434 \pm 0.205$ & $13.6 \pm 18.2$ & $<\mathbf{0 . 0 0 0 1}$ \\
$D_{t}$ & $0.949 \pm 0.153$ & $1.072 \pm 0.184$ & $14.5 \pm 20.8$ & $<\mathbf{0 . 0 0 0 1}$ \\
$\left(10^{-3} \mathrm{~mm}^{2} / \mathrm{s}\right)$ & & & & \\
$f(\%)$ & $15.8 \pm 4.7$ & $16.3 \pm 5.1$ & $8.8 \pm 41.1$ & 0.420 \\
$D^{*}$ & $28.4 \pm 18.1$ & $31.7 \pm 2.1$ & $57.9 \pm 156.5$ & 0.185 \\
$\left(10^{-3} \mathrm{~mm}^{2} / \mathrm{s}\right)$ & & & & \\
$D^{*} \times f$ & $424 \pm 305$ & $466 \pm 227$ & $51.0 \pm 129.1$ & 0.076 \\
$\left(10^{-3} \mathrm{~mm}^{2} / \mathrm{s}\right)$ & & & & \\
\hline
\end{tabular}

${ }^{a}$ Average \pm standard deviation. $P$ values refer to Wilcoxon test. Statistically significant $p$-values are bold. Abbreviations: $A D C$ Apparent diffusion coefficient, $D_{t}$ Tissue diffusion coefficient, $D^{*}$ Perfusion-related diffusion coefficient, $f(\%)$ Perfusion fraction, $D^{*} \times f$ Product of $D^{*}$ by $f$
Table 3 Results of Spearman's correlation tests between parotid shrinkage and imaging/ anthropometric parameters at baseline

\begin{tabular}{|c|c|c|c|c|}
\hline \multirow{2}{*}{$\begin{array}{l}\text { Variable at } \\
\text { baseline }\end{array}$} & \multicolumn{2}{|l|}{$\Delta \mathrm{Vol}_{1 \text { ofr }}(\%)$} & \multicolumn{2}{|l|}{$\Delta \mathrm{Vol}_{\text {post }}(\%)$} \\
\hline & Spearman's Rho & $p$-value & Spearman's Rho & $p$-value \\
\hline$A D C$ & -0.185 & 0.100 & -0.010 & 0.932 \\
\hline $\mathrm{D}_{t}$ & -0.099 & 0.385 & -0.061 & 0.609 \\
\hline$f$ & -0.056 & 0.620 & -0.002 & 0.986 \\
\hline$D^{*}$ & -0.019 & 0.865 & -0.188 & 0.109 \\
\hline $\mathrm{D}^{*} \times f$ & -0.007 & 0.950 & -0.167 & 0.154 \\
\hline$K^{\text {trans }}$ & 0.210 & 0.069 & 0.434 & $<0.001$ \\
\hline$K_{e p}$ & 0.239 & 0.038 & 0.349 & 0.003 \\
\hline$v_{e}$ & 0.025 & 0.832 & 0.174 & 0.143 \\
\hline IAUGC & 0.139 & 0.231 & 0.375 & 0.001 \\
\hline Parotid volume & -0.242 & 0.031 & -0.056 & 0.636 \\
\hline Age & -0.108 & 0.341 & 0.045 & 0.702 \\
\hline Weight & -0.396 & $<0.001$ & -0.135 & 0.253 \\
\hline BMI & -0.368 & 0.001 & -0.127 & 0.281 \\
\hline$D_{\text {mean }}(G y)$ & 0.226 & 0.044 & -0.007 & 0.951 \\
\hline$V_{30}(\%)$ & 0.266 & 0.017 & 0.086 & 0.468 \\
\hline
\end{tabular}

Statistically significant p-values are bold. Abbreviations: $\Delta V_{\text {Vol }}{ }_{10 f r}$ Parotid shrinkage(\%) at the 10 th fraction, $\Delta V_{\text {Vol }}$ post Parotid shrinkage(\%) 8 weeks after $\mathrm{RT}, A D C$ Apparent diffusion coefficient, $D_{t}$ Tissue diffusion coefficient, $D^{*}$ Perfusion-related diffusion coefficient, $f(\%)$ Perfusion fraction, $D^{*} \times f$ Product of $D^{*}$ by $f, \triangle A D C$ ADC variation (\%) relative to the pretreatment value (analogously for the other imaging variables), $K^{\text {trans }}$ Transfer constant between plasma and EES (extravascular extracellular space), $K_{e p}$ Transfer constant between EES and plasma, $v_{e}$ Fractional volume of EES, IAUGC Initial area under gadolinium concentration curve, $B M I$ Body mass index, $D_{\text {mean }}$ Planned mean dose to the parotid gland, $V_{30}(\%)$ Percentage of parotid volume receiving a dose $\geq 30 \mathrm{~Gy}$

A significant relationship was found between the final parotid shrinkage $\Delta \mathrm{Vol}_{\text {post }}$ and $\mathrm{D}_{\mathrm{t}}, f$ and the changes in $\mathrm{D}_{\mathrm{t}}$ and $f$. Moreover, $\Delta \mathrm{Vol}_{\text {post }}$ was strongly related to $\Delta \mathrm{Vol}_{10 \mathrm{fr}}(\mathrm{Rho}=0.471, p<0.001)$.

Finally, changes in $\mathrm{ADC} / \mathrm{D}_{\mathrm{t}}$ were significantly related to $\mathrm{D}_{\text {mean }}\left(p=0.001, p=0.011\right.$, respectively) and $\mathrm{V}_{30}(\%)$ $(\mathrm{p}<0.001, p=0.001$, respectively) (Additional file 1 : Table S3).

\section{Illustrative cases}

Two illustrative cases are presented in Figs. 3 and 4. In Fig. 3, the case of a 68-year-old man affected by a tonsil carcinoma is shown, whose initial weight and BMI were $65.5 \mathrm{Kg}$ and $21.1\left(\mathrm{Kg} / \mathrm{m}^{2}\right)$, respectively. The three serial MR scans documented a substantial gland shrinkage, while the corresponding perfusion maps indicated well perfused parotids at baseline (the average $\mathrm{K}^{\text {trans }}$ and IAUGC of parotids were $0.868 \mathrm{~min}^{-1}$ and 0.404 , respectively). Curves of DW-signal attenuation of parotids at baseline and during RT demonstrated a noticeable increase in tissue water diffusivity, suggesting a considerable decrease in cell density as a consequence of the radiation damage (the average increase in $\mathrm{ADC}$ and $\mathrm{D}_{\mathrm{t}}$ of parotids 

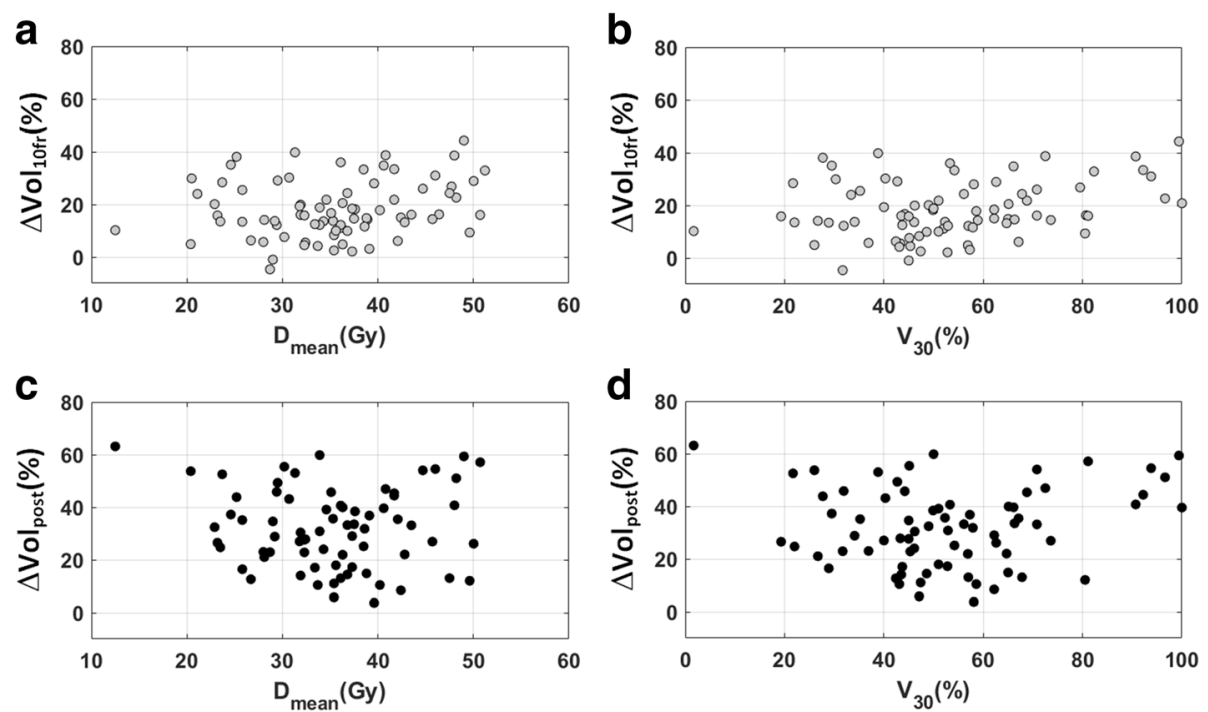

Fig. 2 Scatter plots of parotid shrinkage $(\%)$ at the 10th fraction $\left(\left.\Delta V_{0}\right|_{10 f r}\right)$ versus planned mean dose to the parotid gland $D_{\text {mean }}(\mathbf{a})$ and percentage of parotid volume receiving a dose $\geq 30 \mathrm{~Gy}, V_{30}(\%)(\mathbf{b})$. Analogously, scatter plots of parotid shrinkage(\%) 8 weeks after RT ( $\left.\Delta \mathrm{Vol}_{\text {post }}\right)$ versus $D_{\text {mean }}(\mathbf{c})$ and $V_{30}(\%)(\mathbf{d})$

were $17 \%$ and $33 \%$, respectively). In Fig. 4 , the case of a 48-year-old man affected by a base of the tongue carcinoma is illustrated, whose initial weight and BMI were 97 $\mathrm{Kg}$ and $28.3\left(\mathrm{Kg} / \mathrm{m}^{2}\right)$, respectively. The three serial MR scans showed only a small final parotid shrinkage, while the perfusion maps indicated moderately perfused glands at baseline (the average $\mathrm{K}^{\text {trans }}$ and IAUGC of parotids were $0.276 \mathrm{~min}^{-1}$ and 0.191 , respectively). Correspondingly, a slight difference was observed between the rates at which the DW-signal attenuation of the parotids decreases during RT, suggesting a limited radiation-induced decrease in

Table 4 Results of Spearman's correlation tests between parotid shrinkage and diffusion parameters during treatment

\begin{tabular}{|c|c|c|c|c|}
\hline \multirow{2}{*}{$\begin{array}{l}\text { Variable at } \\
\text { the 10th fraction }\end{array}$} & \multicolumn{2}{|l|}{$\Delta \mathrm{Vol}_{1 \mathrm{ofr}}(\%)$} & \multicolumn{2}{|l|}{$\Delta \mathrm{Vol}_{\text {post }}(\%)$} \\
\hline & Spearman's Rho & $p$-value & Spearman's Rho & $p$-value \\
\hline$A D C$ & 0.121 & 0.285 & 0.103 & 0.383 \\
\hline$D_{t}$ & 0.286 & 0.011 & 0.289 & 0.013 \\
\hline F & -0.057 & 0.614 & -0.232 & 0.047 \\
\hline$D^{*}$ & -0.114 & 0.314 & 0.122 & 0.299 \\
\hline$D^{*} \times f$ & -0.153 & 0.176 & -0.013 & 0.910 \\
\hline$\triangle \mathrm{ADC}$ & 0.228 & 0.042 & 0.173 & 0.141 \\
\hline$\Delta \mathrm{D}_{t}$ & 0.319 & 0.004 & 0.372 & 0.001 \\
\hline$\Delta f$ & 0.022 & 0.844 & -0.242 & 0.038 \\
\hline$\Delta D^{*}$ & -0.117 & 0.301 & 0.192 & 0.101 \\
\hline$\Delta \mathrm{D}^{*} \times f$ & -0.119 & 0.293 & 0.096 & 0.416 \\
\hline
\end{tabular}

Statistically significant $\mathrm{p}$-values are bold. Abbreviations: $\Delta V \mathrm{Vol}_{\text {ofr }}$ Parotid shrinkage $(\%)$ at the 10 th fraction, $\Delta V$ ol $_{\text {post }}$ Parotid shrinkage(\%) 8 weeks after $\mathrm{RT}, A D C$ Apparent diffusion coefficient, $D_{t}$ Tissue diffusion coefficient, $D^{*}$ Perfusion-related diffusion coefficient, $f(\%)$ Perfusion fraction, $D^{*} \times f$ Product of $D^{*}$ by $f, \triangle A D C$ ADC variation (\%) relative to the pretreatment value (analogously for the other imaging variables) cell density (the average increase in $\mathrm{ADC}$ and $\mathrm{D}_{\mathrm{t}}$ of parotids were $9 \%$ and $6 \%$, respectively).

\section{Discussion}

Image-based parameters evaluating early changes of irradiated organs have shown the potential to guide both treatment optimization and personalization, as supported by the increasing number of data published in the last few years $[5,21-26]$. In the present study we investigated whether either IVIM-DWI or DCE-MRI can provide quantitative indices with radiobiological relevance, as tissue cellular density and vascular perfusion, to timely detect and predict parotid gland shrinkage during/after (chemo)radiotherapy for oropharyngeal cancer. In order to maximize the potential benefits of adaptive re-planning for those patients who undergo significant parotid shrinkage, early evaluation of radiation-induced parotid gland modifications, possibly during the first 2 weeks of treatment $[8,27]$, is advised.

Anatomical change of parotid glands during radiotherapy may have dosimetric as well as clinical implications. The former is related to the higher dose received during treatment by the glands that show the larger variations $[7,8]$; the latter to some extent that early anatomical modifications of parotids are associated with a reduction in salivary output, both acute and late [4, 26-28]. However, further investigation is underway to corroborate this point.

We found mean parotid shrinkages, $\Delta \mathrm{Vol}_{10 \mathrm{fr}}$ and $\Delta \mathrm{Vol}_{\text {post }}$, in satisfactory agreement with those reported by other investigators $[7,8]$. Interestingly, we observed relatively similar values for both $\mathrm{ADC}$ and $\mathrm{D}_{t}$ across patients at baseline, suggesting that the glandular tissue is 

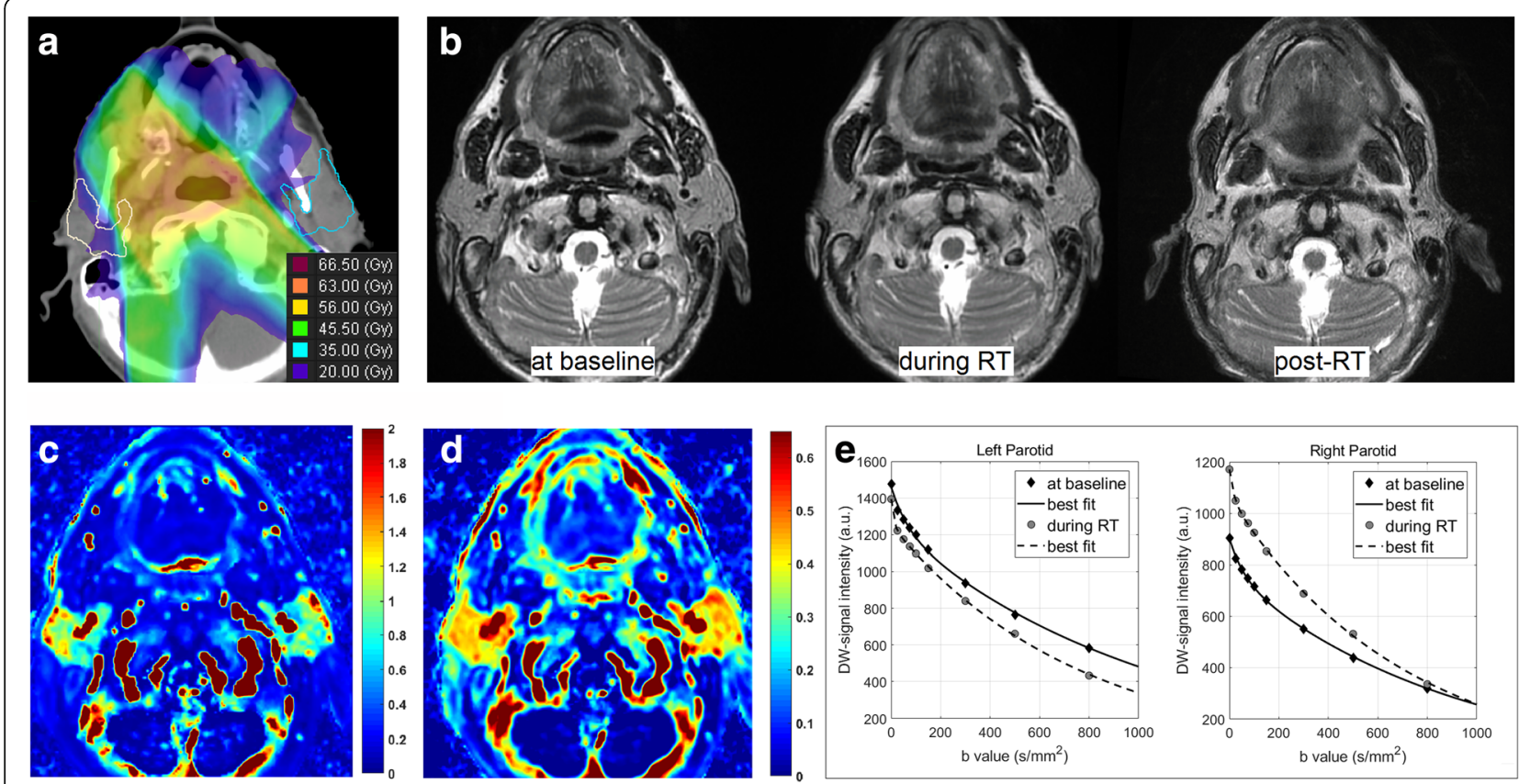

Fig. 3 A 68-year-old man affected by a tonsil carcinoma: dose distribution overlaid on the central section of the parotid glands on axial CT (a); T2-weighted images before, at 2 weeks of radiotherapy and 8 weeks after radiotherapy (b) documenting a substantial gland shrinkage; the corresponding maps of $\mathrm{K}^{\text {trans }}$ in $\min ^{-1}$ (c) and IAUGC in a.u. (d), at baseline, indicating well perfused parotids. Curves of DW-signal attenuation of parotids at baseline and during RT (e): the modification of the DW-signal attenuation curves during RT documents a noticeable increase in tissue water diffusivity, suggesting a considerable decrease in cell density as a consequence of the radiation damage
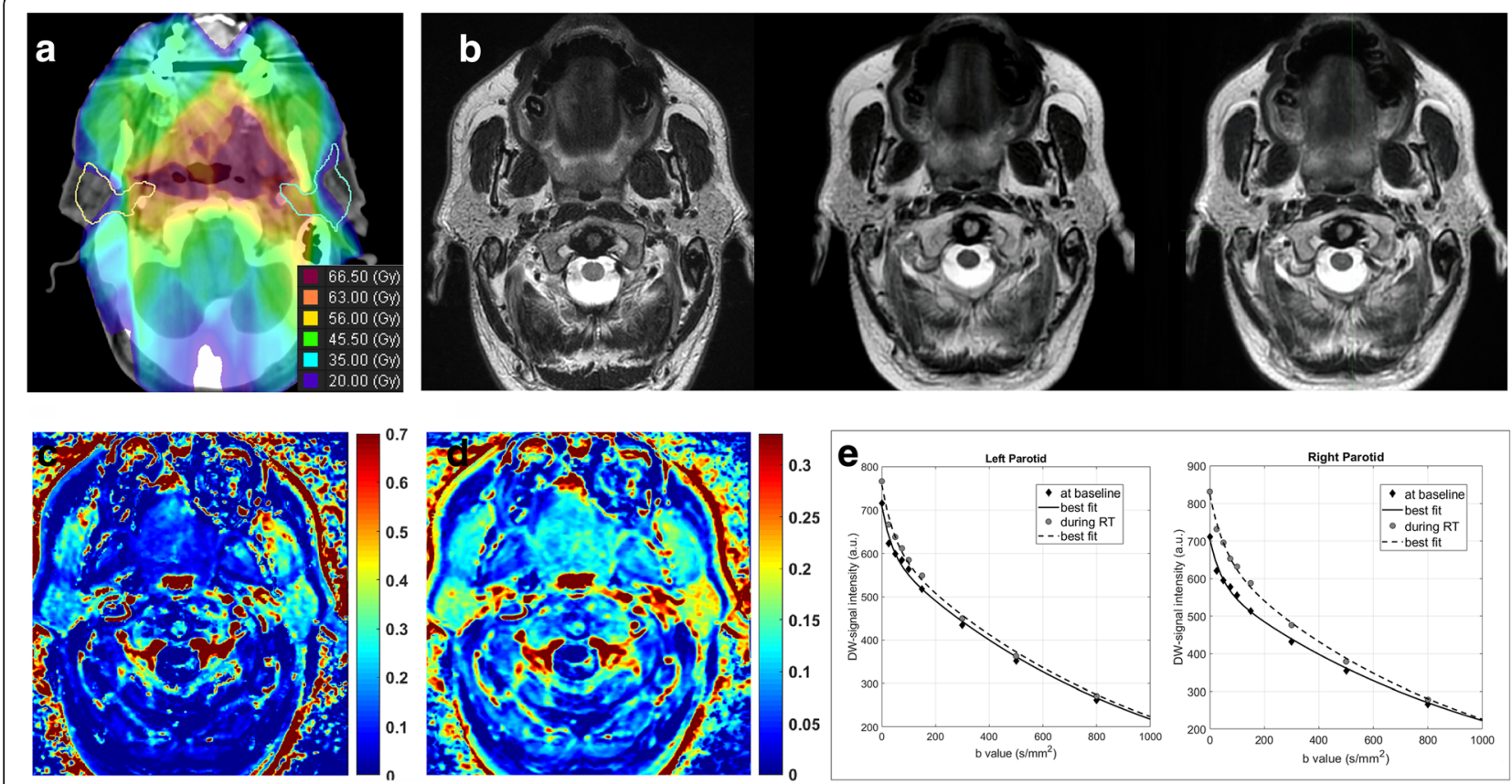

Fig. 4 A 48-year-old man affected by a base of the tongue carcinoma: dose distribution overlaid on the central section of the parotid glands on axial CT (a); T2-weighted images before, at 2 weeks of radiotherapy and 8 weeks after radiotherapy (b) showing a small final parotid shrinkage; the corresponding maps of $\mathrm{K}^{\text {trans }}$ in $\mathrm{min}^{-1}$ (c) and IAUGC in a.u. (d), at baseline, indicating moderately perfused glands. Correspondingly, a slight difference was observed between the rates at which the DW-signal attenuation of the parotids decreases during RT (e) 
remarkably similar in diffusion coefficients in different patients, even if a small but statistically significant positive correlation was found between ADC and age (Additional file 1: Table S1 and Additional file 2: Figure S1). Such limited variability may have contributed to the lack of association between these parameters and parotid shrinkage, both during and after RT. Conversely, DCE-MRI features were highly different across patients at baseline, suggesting highly individual perfusion patterns. Moreover, the inverse relationship between BMI and DCE-MRI parameters (Additional file 1: Table S1 and Additional file 2: Figure S2) suggests a reduced proportional volume of vascular tissue in overweight patients. We also found that hypo-perfused parotids tend to show reduced ADC and $D_{t}$ values, thus a reduced water molecule mobility (Additional file 1: Table S2). One may argue that this is indirectly related to the fact that the adipose tissue limits the mobility of water molecules as shown by Chang et al. [29], though no direct correlation was found between diffusion and BMI in our data. This suggests that the diffusion parameters, especially $A D C$ and $D_{t}$, are not just a simple expression of cellularity but seem to be dependent upon the characteristics of the glandular tissue (i.e. the proportion of glandular versus fatty components). To this end, we show that both diffusion and perfusion imaging may be useful to individually assess/monitor structural and functional properties of parotids.

Selected DCE-MRI parameters, such as $K^{\text {trans }}$ and $K_{\text {ep }}$, showed potential to detect parotid shrinkage both during and early after IMRT: patients with more vascularized parotid glands exhibited a greater volume reduction, possibly due to a higher tissue oxygenation and thus an increased radiosensitivity [5] (see also Figs. 3 and 4). On the other hand, Lee et al. [30] reported an inverse association between parotid shrinkage 3 months after RT for nasopharyngeal cancer and plasma volume derived from DCE-MRI. According to the Authors a higher plasma volume translates into a better perfusion thought it remains unclear how this would lead to an improved protection to radiation damage.

A previous study of Houweling et al. [21] also investigated the role of MRI to quantify the early radiationinduced modifications of the salivary glands in a population of patients with oropharyngeal carcinoma. The Authors documented a strong correlation between the increase in T2-weighted signal from baseline to 6 weeks after RT and the planned mean dose to parotid and submandibular glands. After treatment, they also found a decrease in $k_{\mathrm{ep}}$ and an increase in $\mathrm{v}_{\mathrm{e}}$ which have been explained by a presumed rise in water content due to vascular edema. Similarly, we found an increase in ADC and $D_{t}$, suggesting a larger extra-cellular/extra-vascular space allowing a higher tissue water mobility.

Initial BMI and patient weight were significantly correlated to $\Delta \mathrm{Vol}_{10 \mathrm{fr}}$ consistent with a greater fatty component resulting in a smaller gland shrinkage, suggesting that in the first few weeks of treatment the glandular tissue is targeted preferentially over the adipose one [22]. The inverse relationship between parotid volume at baseline and $\Delta \mathrm{Vol}_{10 \text { fr }}$ likely reflects the strong positive correlation between the PG volume at baseline and both BMI and patient weight.

Parotid shrinkage at 2 weeks was found to be weakly related to both PG $D_{\text {mean }}$ and $V_{30}$, suggesting that the radiation-induced loss of acinar cells is a dose-dependent effect as previously shown $[8,23]$. Accordingly, we found $D_{\text {mean }}$ and $V_{30}$ to be correlated to changes of $A D C / D_{t}$ during the first 2 weeks of treatment that are presumed to reflect variations of cellular density (Additional file 1: Table S3). Moreover, at 2 weeks, the perfusion-free diffusion coefficient, $D_{t}$, was found to be more predictive of PG shrinkage than ADC at either checkpoint (Table 4) suggesting a higher sensitivity in variations in cellular microstructure/depopulation than ADC [23] which is susceptible to the effects of both perfusion and diffusion [15]. The present data are also in agreement with the association between parotid shrinkage and parotid density reduction (acinar cell depopulation and relative increase in fibrofatty tissue) observed in CT-based studies [22].

Similarly to others [9], we could not find an association between parotid shrinkage after radiotherapy and both $D_{\text {mean }}$ and $V_{30}$, while a correlation was present after the first 2 weeks of treatment. There are multiple possible explanations for this change in predictive power of dose: progressive deterioration of the dose distribution throughout the course of treatment; delivery of a threshold dose above which parotid shrinkage is minimal; other confounding factors, such as weight loss or variability in parotid gland composition [7-9, 21, 27]. Conversely, earlier during treatment, shrinkage is likely to reflect more closely the depopulation of acinar cells, and thus, dose may play a more prominent and detectable role $[8,27]$. Moreover, the limited variance in dose values and the small sample size may have contributed to mask an effect of dose on parotid shrinkage after treatment.

It is unclear why $\Delta \mathrm{Vol}_{\text {post }}$ was related to in-treatment $f$ values and changes in $f$, as no significant association was observed between initial perfusion-related diffusion coefficients $\left(\mathrm{D}^{*}, \mathrm{f}\right.$ and $\left.\mathrm{D}^{*} \times f\right)$ and any DCE-MRI perfusion parameters. However, contradictory results on the ability of IVIM-DWI in assessing tissue perfusion have been reported in the literature to date [17]. This may reflect either the presence of confounding sources of incoherent motion in biological tissues and/or the difficulty to obtain an optimal image quality with minimal motion and susceptibility artifacts, particularly in patients with head and neck cancer $[15,17]$.

The study has some limitations. We extracted the median value of the signal from all the voxels within the entire 
gland as opposed to the punctual 'local' signal from discrete sub-regions of the PG. We also did not investigate the ability of MRI to detect a different local effect of radiation dose, as suggested by others [21]; this may help to identify sub-regions with different radiosensitivity within the gland [31]. On the other hand, we believe that our approach allows the extraction of data less susceptible to motion artifacts and image co-registration issues and ultimately more consistent. Future investigations from this on-going study will include both patient-reported xerostomia as an endpoint as soon as clinical data mature and a multivariable model on selected quantitative image analyses, in addition to patient- and treatment-related covariates.

\section{Conclusions}

Both IVIM-DWI and DCE-MRI can help to detect early (during treatment) as well as late (after treatment completion) PG shrinkage. Moreover, they shed light upon the complex correlation between the volumetric change of PG and patient-/treatment-related variables by assessing individual microcapillary perfusion and tissue diffusivity.

\section{Additional files}

Additional file 1: Table S1. Spearman's Rho values between DCE-MRI/ IVIM-DWI at baseline and anthropometric variables at baseline. Table S2. Spearman's Rho values between DCE-MRI and IVIM-DWI parameters at baseline. Table S3. Spearman's Rho values among changes of IVIM-DWI parameters during treatment and dosimetric variables. (DOCX $22 \mathrm{~kb}$ )

Additional file 2: Figure S1. Scatter plots of IVIM-DWI parameters versus Age and BMI. Figure S2. Scatter plots of DCE-MRI parameters versus Age and BMI. (ZIP $212 \mathrm{~kb})$

\section{Abbreviations \\ ADC: Apparent diffusion coefficient; BMl: Body mass index; $D^{*} \times f$ : Product of $D^{*}$ by $f ; D^{*}$ : Perfusion-related diffusion coefficient; $D_{\text {mean }}$ : Planned mean dose to the parotid gland; $\mathrm{D}_{\mathrm{t}}$ : Tissue diffusion coefficient; EES: Extravascular extracellular space; $f(\%)$ : Perfusion fraction; IAUGC: Initial area under gadolinium concentration curve; IMRT: Intensity modulated radiotherapy; $K_{\text {ep: }}$ Transfer constant between EES and plasma; $K^{\text {trans }}$ : Transfer constant between plasma and EES; PG: Parotid gland; $V_{30}(\%)$ : Percentage of parotid volume receiving a dose $\geq 30 \mathrm{~Gy}$; $v_{\mathrm{e}}$ : Fractional volume of EES; $\triangle \mathrm{Vol}_{1 \text { ofr: }}$ : Parotid shrinkage(\%) at the 10th fraction; $\triangle A D C$ : ADC variation (\%) relative to the pretreatment value (analogously for the other imaging variables); $\Delta$ Vol $_{\text {post: }}$ Parotid shrinkage(\%) 8 weeks after RT}

\section{Acknowledgements}

The authors are indebted to Michele Farella and to Pierfrancesco Rinaldi for their continued technical assistance.

\section{Funding}

This work was supported by the Italian Association for Cancer Research (AIRC, project No.17028)

\section{Availability of data and materials}

The datasets used and/or analyzed during the current study are available from the corresponding author on reasonable request.

\section{Authors' contributions}

SM and GS conceived of the study and drafted the manuscript. LM and AF contributed to the patient enrollment and data gathering. AV was responsible for the MRI scans and participated in the design of the study. FD carried out the image delineation and participated in the image analysis. FS carried out the responsibility of collecting and maintaining the data. IT performed the statistical analysis. All authors read and approved the final manuscript.

Ethics approval and consent to participate

This study was approved by the research ethics committee of our Institute.

Consent for publication

Patients gave written consent for data collection and analysis.

\section{Competing interests}

The authors declare that they have no competing interests.

\section{Publisher's Note}

Springer Nature remains neutral with regard to jurisdictional claims in published maps and institutional affiliations.

\section{Author details}

${ }^{1}$ Medical Physics Laboratory, IRCCS Regina Elena National Cancer Institute, Via Elio Chianesi 53, 00144 Rome, Italy. ${ }^{2}$ Department of Radiotherapy, IRCCS Regina Elena National Cancer Institute, Via Elio Chianesi 53, 00144 Rome, Italy. ${ }^{3}$ Radiology and Diagnostic Imaging Department, IRCCS Regina Elena National Cancer Institute, Via Elio Chianesi 53, 00144 Rome, Italy. ${ }^{4}$ Department of Biomedicine and Prevention, University of Rome "Tor Vergata", Viale Oxford 81, 00133 Rome, Italy. ${ }^{5}$ Biostatistics-Scientific Direction, IRCCS Regina Elena National Cancer Institute, Via Elio Chianesi 53, 00144 Rome, Italy.

Received: 23 April 2018 Accepted: 24 September 2018

Published online: 01 October 2018

\section{References}

1. Pfister DG, Ang KK, Brizel DM, Burtness BA, Cmelak AJ, Colevas AD, Dunphy F, Eisele DW, Gilbert J, Gillison ML, Haddad RI, Haughey BH, Hicks WL Jr, Hitchcock YJ, Kies MS, Lydiatt WM, Maghami E, Martins R, McCaffrey T, Mittal BB, Pinto HA, Ridge JA, Samant S, Sanguineti G, Schuller DE, Shah JP, Spencer S, Trotti A 3rd, Weber RS, Wolf GT, Worden F, National Comprehensive Concer Network. Head and neck cancers. J Natl Compr Cancer Netw. 2011;9:596-650.

2. Hawkins PG, Kadam AS, Jackson WC, Eisbruch A. Organ-sparing in radiotherapy for head-and-neck Cancer: improving quality of life. Semin Radiat Oncol. 2018;28:46-52.

3. Langendijk JA, Doornaert P, Verdonck-de Leeuw IM, Leemans CR, Aaronson NK, Slotman BJ. Impact of late treatment-related toxicity on quality of life among patients with head and neck cancer treated with radiotherapy. J Clin Oncol. 2008;26:3770-6.

4. van Dijk LV, Brouwer CL, van der Laan HP, Burgerhof JGM, Langendijk JA, Steenbakkers RJHM, Sijtsema NM. Geometric image biomarker changes of the parotid gland are associated with late Xerostomia. Int J Radiat Oncol Biol Phys. 2017:99:1101-10.

5. van Dijk LV, Brouwer CL, van der Schaaf A, Burgerhof JGM, Beukinga RJ, Langendijk JA, Sijtsema NM, Steenbakkers RJHM. CT image biomarkers to improve patient-specific prediction of radiation-induced xerostomia and sticky saliva. Radiother Oncol. 2017;122:185-91.

6. Meirovitz A, Murdoch-Kinch CA, Schipper M, Pan C, Eisbruch A. Grading xerostomia by physicians or by patients after intensity-modulated radiotherapy of head-and-neck cancer. Int J Radiat Oncol Biol Phys. 2006;66:445-53.

7. Barker JL Jr, Garden AS, Ang KK, O'Daniel JC, Wang H, Court LE, Morrison WH, Rosenthal DI, Chao KS, Tucker SL, Mohan R, Dong L. Quantification of volumetric and geometric changes occurring during fractionated radiotherapy for head-and-neck cancer using an integrated CT/linear accelerator system. Int J Radiat Oncol Biol Phys. 2004;59:960-70.

8. Zhang Y, Lin C, Wu J, Jiang X, Lee SWY, Tam SY, Wu WWC. A longitudinal evaluation of early anatomical changes of parotid gland in intensity modulated radiotherapy of nasopharyngeal carcinoma patients with parapharyngeal space involvement. J Med Radiat Sci. 2017;64:188-94.

9. Sanguineti G, Ricchetti F, Thomas O, Wu B, McNutt T. Pattern and predictors of volumetric change of parotid glands during intensity modulated radiotherapy. Br J Radiol. 2013;86:20130363.

10. Wang W, Lang J. Strategies to optimize radiotherapy based on biological responses of tumor and normal tissue. Exp Ther Med. 2012;4:175-80. 
11. Caudell JJ, Torres-Roca JF, Gillies RJ, Enderling H, Kim S, Rishi A, Moros EG, Harrison LB. The future of personalised radiotherapy for head and neck cancer. Lancet Oncol. 2017;18:e266-73.

12. Wong KH, Panek R, Bhide SA, Nutting CM, Harrington K, Newbold KL. The emerging potential of magnetic resonance imaging in personalizing radiotherapy for head and neck cancer: an oncologist's perspective. $\mathrm{Br}$ J Radiol. 2017;90:20160768.

13. Cao $Y$. The promise of dynamic contrast-enhanced imaging in radiation therapy. Semin Radiat Oncol. 2011;21:147-56.

14. Tofts PS, Brix G, Buckley DL, Evelhoch JL, Henderson E, Knopp MV, Larsson HB, Lee TY, Mayr NA, Parker GJ, Port RE, Taylor J, Weisskoff RM. Estimating kinetic parameters from dynamic contrast-enhanced T(1)-weighted MRI of a diffusable tracer: standardized quantities and symbols. J Magn Reson Imaging. 1999;10:223-32

15. Taouli B, Beer AJ, Chenevert T, Collins D, Lehman C, Matos C, Padhani AR, Rosenkrantz AB, Shukla-Dave A, Sigmund E, Tanenbaum L, Thoeny $H$, Thomassin-Naggara I, Barbieri S, Corcuera-Solano I, Orton M, Partridge SC, Koh DM. Diffusion-weighted imaging outside the brain: consensus statement from an ISMRM-sponsored workshop. J Magn Reson Imaging. 2016;44:521-40.

16. Le Bihan D, Breton E, Lallemand D, Aubin ML, Vignaud J, Laval-Jeantet M. Separation of diffusion and perfusion in intravoxel incoherent motion MR imaging. Radiology. 1988;168:497-505.

17. Federau C. Intravoxel incoherent motion MRI as a means to measure in vivo perfusion: a review of the evidence. NMR Biomed. 2017;30. https://doi.org/ 10.1002/nbm.3780 Epub 2017 Sep 8.

18. Sanguineti G, Gunn GB, Endres EJ, Chaljub G, Cheruvu P, Parker B. Patterns of locoregional failure after exclusive IMRT for oropharyngeal carcinoma. Int J Radiat Oncol Biol Phys. 2008;72:737-46.

19. Fedorov A, Beichel R, Kalpathy-Cramer J, Finet J, Fillion-Robin JC, Pujol S, Bauer C, Jennings D, Fennessy F, Sonka M, Buatti J, Aylward S, Miller JV, Pieper S, Kikinis R. 3D slicer as an image computing platform for the quantitative imaging network. Magn Reson Imaging. 2012;30:1323-41.

20. Marzi S, Piludu F, Vidiri A. Assessment of diffusion parameters by intravoxe incoherent motion MRI in head and neck squamous cell carcinoma. NMR Biomed. 2013;26:1806-14.

21. Houweling AC, Schakel T, van den Berg CA, Philippens ME, Roesink JM, Terhaard CH, Raaijmakers CP. MRI to quantify early radiation-induced changes in the salivary glands. Radiother Oncol. 2011;100:386-9.

22. Fiorino C, Rizzo G, Scalco E, Broggi S, Belli ML, Dell'Oca I, Dinapoli N, Ricchetti F, Rodriguez AM, Di Muzio N, Calandrino R, Sanguineti G, Valentini V , Cattaneo GM. Density variation of parotid glands during IMRT for headneck cancer: correlation with treatment and anatomical parameters. Radiother Oncol. 2012;104:224-9.

23. Marzi S, Forina C, Marucci L, Giovinazzo G, Giordano C, Piludu F, Landoni V, Spriano G, Vidiri A. Early radiation-induced changes evaluated by intravoxel incoherent motion in the major salivary glands. J Magn Reson Imaging. 2015;41:974-82.

24. Joint Head and Neck Radiotherapy-MRI Development Cooperative. Dynamic contrast-enhanced MRI detects acute radiotherapy-induced alterations in mandibular microvasculature: prospective assessment of imaging biomarkers of normal tissue injury. Sci Rep. 2016;6:29864.

25. Zhou N, Chu C, Dou X, Li M, Liu S, Zhu L, Liu B, Guo T, Chen W, He J, Yan J, Zhou Z, Yang X, Liu T. Early evaluation of irradiated parotid glands with intravoxel incoherent motion MR imaging: correlation with dynamic contrast-enhanced MR imaging. BMC Cancer. 2016;16:865-016-2900-2.

26. Zhou N, Guo T, Zheng H, Pan X, Chu C, Dou X, Li M, Liu S, Zhu L, Liu B, Chen W, He J, Yan J, Zhou Z, Yang X. Apparent diffusion coefficient histogram analysis can evaluate radiation-induced parotid damage and predict late xerostomia degree in nasopharyngeal carcinoma. Oncotarget. 2017:8:70226-38.

27. Belli ML, Scalco E, Sanguineti G, Fiorino C, Broggi S, Dinapoli N, Ricchetti F, Valentini V, Rizzo G, Cattaneo GM. Early changes of parotid density and volume predict modifications at the end of therapy and intensity of acute xerostomia. Strahlenther Onkol. 2014;190:1001-7.

28. Teshima K, Murakami R, Tomitaka E, Nomura T, Toya R, Hiraki A, Nakayama H, Hirai T, Shinohara M, Oya N, Yamashita Y. Radiation-induced parotid gland changes in oral cancer patients: correlation between parotid volume and saliva production. Jpn J Clin Oncol. 2010;40:42-6.

29. Chang HC, Juan CJ, Chiu HC, Cheng CC, Chiu SC, Liu YJ, Chung HW, Hsu $\mathrm{HH}$. Effects of gender, age, and body mass index on fat contents and apparent diffusion coefficients in healthy parotid glands: an MRI evaluation. Eur Radiol. 2014;24:2069-76.

30. Lee FK, King AD, Kam MK, Ma BB, Yeung DK. Radiation injury of the parotid glands during treatment for head and neck cancer: assessment using dynamic contrast-enhanced MR imaging. Radiat Res. 2011;175:291-6.

31. van Luijk P, Pringle S, Deasy JO, Moiseenko W, Faber H, Hovan A, Baanstra M, van der Laan HP, Kierkels RG, van der Schaaf A, Witjes MJ, Schippers JM, Brandenburg S, Langendijk JA, Wu J, Coppes RP. Sparing the region of the salivary gland containing stem cells preserves saliva production after radiotherapy for head and neck cancer. Sci Transl Med. 2015;7:305ra147.
Ready to submit your research? Choose BMC and benefit from:

- fast, convenient online submission

- thorough peer review by experienced researchers in your field

- rapid publication on acceptance

- support for research data, including large and complex data types

- gold Open Access which fosters wider collaboration and increased citations

- maximum visibility for your research: over $100 \mathrm{M}$ website views per year

At BMC, research is always in progress.

Learn more biomedcentral.com/submissions 